\title{
'||||||||||||||||||||||||||||||||||||||||||||||||||||||||||||||||||||.
}

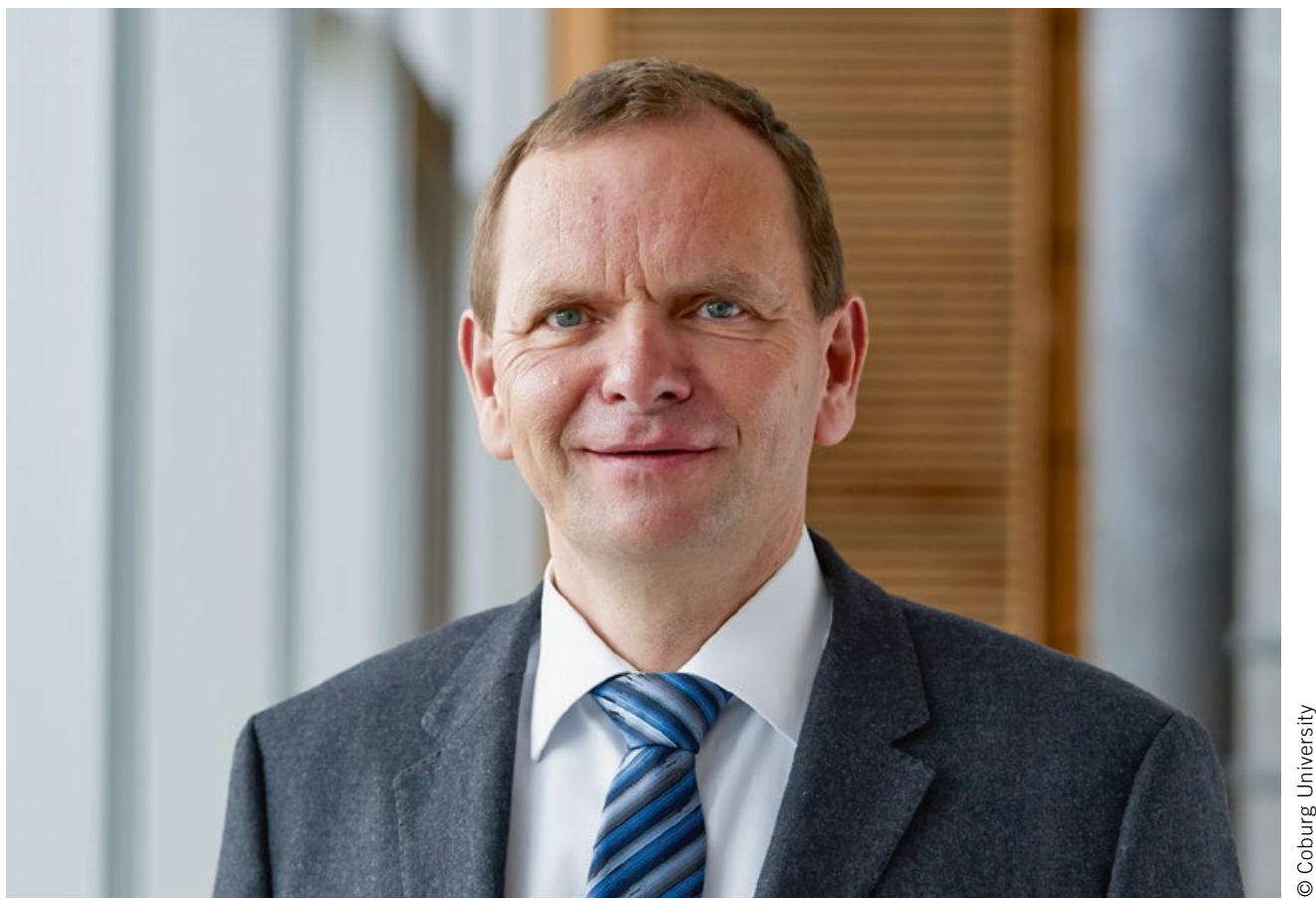

Prof. Dr. Jürgen Krahl Spokesman of the Board of the Technology Transfer Center Automotive at Coburg University (TAC)

\section{Perfecting the Triad}

Ever since the internal combustion engine was invented, the focus of engine development has been on achieving the most efficient conversion of chemical energy into kinetic energy. With the introduction of the first emissions standards in the 1960s, it was recognised that the composition of the fuel had considerable influence on combustion, and therefore on emissions. In the 1980s, tetraethyl lead was replaced by methyl tert-butyl ether (MTBE) in order to counteract the negative impact of lead compounds on the environment.

Form today's perspective, the introduction of unleaded gasoline can be seen as an early example of the interaction between engine technology and chemistry. Since the 1990s, the pressure of increasingly strict emissions legislation has led to a reduction in aromatic and sulphur compounds in fuels. Among other things, this step was required in order to improve the long-term functionality of exhaust aftertreatment systems. Compared to the rapid further development of engines and exhaust aftertreatment over the past 20 years, the fuels themselves have changed only slightly so far. We can therefore assume that fuels have not yet developed their full potential in the fuel - engine - exhaust aftertreatment triad.

Currently, discussions are focused on how best to comply with $\mathrm{CO}_{2}$ legislation. The diesel engine is additionally in the public and technical spotlight due to its $\mathrm{NO}_{\mathrm{x}}$ emissions. On the one hand, the addition of bio-components such as biodiesel or hydrotreated vegetable oil (HVO) can help to improve the envi- ronmental impact of climate-changing gases. On the other hand, increased $\mathrm{NO}_{\mathrm{x}}$ emissions were observed for $\mathrm{HVO}$ and particularly for biodiesel. In the medium term, further research is required in this field, and this research will become increasingly important especially in the context of an expected global rise in resource diversification and the simultaneous demand for drop-in fuels. These are alternative fuels that will be able to replace conventional ones without technical modifications and which are fully blendable with them.

An analogue situation exists for direct-injection sparkignition engines, which have a known tendency to form particles. The simultaneous optimisation of both the fuel and the combustion process will offer the fundamental opportunity to comply with emissions standards even without a gasoline particle filter.

Fuel is a key component for engines even today, and it can only be successfully optimised in harmony with the engine and the exhaust aftertreatment system.

Therefore, in addition to the study of pollutant formation, the consideration of fuel as a source of energy must also involve an approach that sees both fuels and exhaust components as chemicals which react with each other and which can trigger desirable or undesirable effects. Fuel chemistry develops the duality of engine combustion and exhaust aftertreatment to form a triad in the sense of a parameter expansion. 